\title{
COMMENTARY
}

\section{The Physician Workforce and Counting What Counts in Primary Care}

\author{
Eugene C. Rich, MD
}

(Fam Med. 2018;50(8):579-82.)

doi: 10.22454/FamMed.2018.595198

$\mathbf{T}$ he US debate over the primary care workforce began in the 1960s with the collapse of medical student interest in general practice and the growth in specialty training. This led to the Millis Commission report on graduate medical education that identified the need for the "primary physician" to "serve as the primary medical resource and counselor to an individual or a family." Nonetheless, even current workforce analyses reflect ongoing confusion regarding what primary care is, and which clinicians can provide it. This problem may contribute to conflicting assessments in several recent reports of the current and future workforce needs in primary care..$^{2-4}$

The Millis report offered additional specific requirements of the primary care physician, such as "understanding and treating the whole man [sic]"; "continuing and comprehensive responsibility"; and "see that the necessary arrangements are made" for hospital or specialist physician services. In a recent review, O'Malley et al reaffirmed the continuing relevance of the core elements invoked by the Millis Commission that collectively differentiate primary care from other clinician services. These are "first-contact accessibility, continuity, comprehensiveness, coordination, and accountability for the whole person. ${ }^{.5}$ Of course it is not one element alone that achieves the primary care role envisioned in the Millis report. Neighborhood retail clinics are indeed accessible; emergency room physicians combine accessibility with comprehensive services, and hospitalist physicians retain comprehensive skills while coordinating with other clinicians to meet urgent patient needs. Both Millis and O'Malley make clear that it is not general training that characterizes primary care; it is the clinician who delivers all the features that provides primary care.

Nonetheless some current workforce analyses continue to conflate training background with primary care practice. For example, some commentators arguing no shortage in primary care $^{3}$ cite the Kaiser Family Foundation (KFF) report of over 456,000 US "primary care physicians."' However KFF includes 188,000 internal medicine physicians - many of whom are in hospitalist or other specialized roles. KFF also includes 51,000 obstetrics and gynecology physicians whose training is not designed to assure comprehensive skills in managing the multiple chronic medical conditions common in adult medicine today. The recent Association of American Medical Colleges (AAMC)-sponsored assessment of physician supply and demand narrows the relevant generalist disciplines to family medicine, general internal medicine, general pediatrics, and geriatric medicine and also excludes 28,000 of these physicians who chiefly provide hospital-based services. With this narrower set of disciplines and practice settings, the report estimates 2016 primary

From the Mathematica Policy Research Center on Health Care Effectiveness, Washington, DC. 
care physician supply much more conservatively at around 240,000, and estimates a current shortage of nearly $14,000 .^{4}$

Another aspect of this debate focuses on the work capacity available in primary care. Some analyses estimate primary care physician workload based on current medical practice data. ${ }^{3}$ Such calculations overlook the constraints of the mispriced, and visit-driven Medicare fee schedule known to discourage achievement of the core features of primary care. ${ }^{7}$ Furthermore, these analyses do not take into account the longstanding evidence that generalist physicians have no time for primary care due to increasing patient complexity and growing diversity of relevant tests and treatments. ${ }^{8}$ Of course not all the services delivered in primary care demand the many years of postbaccalaureate education inherent to generalist physician training. Accordingly various primary care transformation initiatives are underway to discern what configurations of information infrastructure and multidisciplinary teams can efficiently and effectively support generalist physicians in delivering all the core features of primary care to a population of patients.

Continuity is one of these features where the concept of the team offers both potential aids as well as challenges to physician productivity and to effective primary care. It is a rare physician who is available $24 / 7$ to ensure continuing engagement with patients' evolving conditions and health system contacts. Thus a team of clinician partners can allow the patient's personal physician to enjoy nights and weekends off; during working hours, nurses or advanced practice clinician (APC) teammates can address many patient questions, and meet other health care needs. Thus the enhanced information infrastructure and multidisciplinary teams facilitated by primary care payment reform can assure continuity of patient information within the practice. However each contact by a primary care teammate is a moment when the patient's primary physician has not heard first-hand the patient's concerns, observed the clinical findings, synthesized all the information into an assessment and plan. It is currently unclear whether it is this individual "interpersonal continuity" or the team's "informational continuity" which matter most in primary care. Evidence abounds that simply having the clinical information "in the record" does not assure the clinical decision maker will recognize and apply this information at the point of care; and the inefficiencies induced by simple patient handoffs, even between clinical partners, have been recognized for decades. ${ }^{9}$ More research is needed to clarify which primary care patients and problems demand interpersonal continuity for effective care, and when proper use of teammates and electronic health record data will be sufficient. Interestingly, a recent proposal for Medicare payment reform cites emerging evidence of substantial savings that can be achieved when a single generalist physician assumes continuing responsibility for both outpatient and inpatient care for a subgroup of previously hospitalized patients. ${ }^{10}$

Restoring clinician responsibility for management of their hospitalized patients invokes the feature of primary care most demanding of deep clinical skills-comprehensiveness. Although this feature has been noted since the Millis Commission report, the emphasis on comprehensiveness in US primary care has been limited, definitions vague, and evidence sparse. ${ }^{11}$ Not surprisingly there are various signs that primary care physician comprehensiveness has been on the wane in the United States, and some advocates and analyses have suggested that clinicians with much less training and plausibly a narrower set of competencies can successfully fulfill the primary care role. ${ }^{12}$ However new evidence is emerging suggesting that comprehensiveness may be an important feature of the primary care clinician role. Bazemore et al have found that when family physicians provide their patients a broader range of services, patients experience lower costs and fewer hospitalizations. ${ }^{13}$ O'Malley has recently confirmed that general internal medicine and family physicians also vary substantially in the degree of management of their patients' health problems (both chronic and new). Furthermore there is preliminary evidence that when these physicians address the breadth and depth of their patients' problems, patients may experience fewer emergency room visits and lower total costs of care. ${ }^{14}$

The primary care feature of comprehensiveness is highly relevant to workforce projections. In the AAMC-sponsored analysis, the projected future shortfall in primary care physicians is substantially ameliorated by just moderate additional reliance on APCs. AAMC estimates that high use of APCs in primary care could 
result in a surplus of primary care physicians by $2030 .{ }^{4}$ Such analyses imply that the substantially greater time in training required for primary care physicians (and attendant competencies relevant to more comprehensive care) may not be beneficial for many primary care patients. However various studies have suggested that experience is an important aspect of clinical competence across a range of clinical skills from diagnostic expertise to performance of procedures and management of inpatient conditions..$^{15}$ Of course the clinical expertise conferred by the generalist physicians' additional training may not be relevant to the care of some common health concerns, and a number of studies have suggested APCs deliver care of at least equivalent quality to that of primary care physicians in some settings. ${ }^{12}$ While the competencies conferred by the training of APCs may be sufficient to address the immediate needs of some primary care patients, APC training may not assure competence with other problems, like the diagnosis and management of the acute medical illnesses of hospitalized patients.

Inevitably the types of comprehensive competencies relevant to individual patient needs will vary across time and circumstance. The healthy college student may want preventive care and efficient management of common health concerns, but can become the middleaged adult with diabetes, hypertension, and depression. This person may transition to the senior with heart failure, renal insufficiency, and inpatient care needs, and ultimately the homebound frail elder. Over time this patient will benefit from the broad range of competencies needed to deliver comprehensive primary care. A more systematic understanding of what competencies are required for high quality comprehensive care for different patients will be important to better understanding the training required for each of the primary care disciplines. Better understanding is also needed regarding the benefits and risks of moving patients between primary care settings to optimally address changing competency needs for comprehensive care. Perhaps primary care clinicians with sufficient baseline competencies and point-of-care decision support can maintain a continuing relationship with patients throughout these transitions, while providing the needed comprehensiveness of care. ${ }^{16}$
Thus counts of generalist clinicians can tell us nothing about current access to the complete package that is true primary care. Likewise past observations of the productivity of generalist clinicians in existing ambulatory care settings will be uninformative regarding what workforce will be required to provide effective primary care to an aging population with an expanding array of diagnostic and therapeutic options. Further research is urgently needed to answer key questions regarding which primary care features matter most for which patients, and which configurations of teams, clinician competencies, and other resources will be most effective. Only then can we understand the composition of the clinician workforce required to achieve accessible, continuous, comprehensive, coordinated, and accountable care.

CORRESPONDING AUTHOR: Address correspondence to Dr Eugene Rich, Center on Health Care Effectiveness, Mathematica Policy Research, 1100 First Street NE, Washington, DC 20002. 202-250-3544. ERiche@mathematica-mpr.com.

\section{References}

1. Millis JS, Wiggins WS, Wolfe D, et al. The Graduate Education of the Physicians: The Report of the Citizens Commission on Graduate Medical Education. Chicago: American Medical Association; 1966.

2. Kirch DG, Petelle K. Addressing the physician shortage: the peril of ignoring demography. JAMA. 2017;317(19):19471948.

3. Gudbranson E, Glickman A, Emanuel EJ. Reassessing the data on whether a physician shortage exists. JAMA. 2017;317(19):1945-1946.

4. Dall T, West T, Chakrabarti R, Reynolds R, Iacobucci W. 2018 Update: The complexities of physician supply and demand: projections from 2016 to 2030. Washington, DC: Association of American Medical Colleges; 2018. https://aamcblack.global.ssl.fastly.net/production/media/filer_public/85/ d7/85d7b689-f417-4ef0-97fb-ecc129836829/aamc_2018_workforce_projections_update_april_11_2018.pdf. Accessed: May $18,2018$.

5. O'Malley AS, Rich EC, Maccarone A, DesRoches CM, Reid RJ. Disentangling the Linkage of Primary Care Features to Patient Outcomes: A Review of Current Literature, Data Sources, and Measurement Needs. J Gen Intern Med. 2015;30(S3)(suppl 3):S576-S585.

6. Kaiser Family Foundation. Primary care physicians by field. October 2017. http://kff.org/other/state-indicator/primarycare-physicians-by-field/?currentTimeframe. Accessed April 20, 2018.

7. Berenson RA, Rich EC. US approaches to physician payment: the deconstruction of primary care. J Gen Intern Med. 2010;25(6):613-618.

8. Østbye T, Yarnall KS, Krause KM, Pollak KI, Gradison M, Michener JL. Is there time for management of patients with chronic diseases in primary care? Ann Fam Med. 2005;3(3):209-214. 
9. Lofgren RP, Gottlieb D, Williams RA, Rich EC. Post-call transfer of resident responsibility: its effect on patient care. J Gen Intern Med. 1990;5(6):501-505.

10. The University of Chicago Medicine. The Comprehensive Care Physician Payment Model. https://aspe.hhs.gov/system/ files/pdf/255906/ProposalUniversityofChicagoMedicine.pdf. Accessed May 18, 2018.

11. O’Malley AS, Rich EC. Measuring comprehensiveness of primary care: challenges and opportunities. J Gen Intern Med. 2015;30(S3)(suppl 3):S568-S575.

12. Mafi JN, Landon BE. Comparing use of low-value health care services among U.S. advanced practice clinicians and physicians. Ann Intern Med. 2017;166(1):77.

13. Bazemore A, Petterson S, Peterson LE, Phillips RL Jr. More comprehensive care among family physicians is associated with lower costs and fewer hospitalizations. Ann Fam Med. 2015;13(3):206-213.
14. O'Malley AS, Rich EC, et al. Measuring the comprehensiveness of primary care: early measure development and findings from the comprehensive primary care initiative. Academy Health Annual Research meeting. Seattle, WA, June 2018.

15. Rich EC. Advanced practice clinicians and physicians in primary care: still more questions than answers. Ann Intern Med. 2016;165(4):290-291.

16. Rich E, Lipson D, Libersky J, Parchman M. Coordinating Care for Adults With Complex Care Needs in the PatientCentered Medical Home: Challenges and Solutions. White Paper. Rockville, MD: Agency for Healthcare Research and Quality. Published January 2012. 